\title{
Aboriginal health: agreement between general practitioners and patients on their health risk status and screening history
}

\author{
Jessica M. Stewart, ${ }^{1}$ Rob Sanson-Fisher, ${ }^{1}$ Sandra Eades, ${ }^{2}$ Catherine D'Este ${ }^{3}$
}

$\mathrm{M}$ ore than sixty per cent $(62 \%)$ of the health disparity between Aboriginal and Torres Strait Islander people and non-Indigenous Australians has been attributed to only six health risk factors: smoking, high body mass, physical inactivity, high cholesterol, high blood pressure and alcohol consumption. ${ }^{1}$ Primary health care plays an important role in addressing these health issues through accurate and timely screening of health risks and in providing appropriate counselling to encourage behavioural change. ${ }^{2}$ Yet many people do not receive the appropriate interventions that are recommended by evidence-based guidelines for clinical prevention., ${ }^{3,4}$ For example, the recent Care Track study found that only $57 \%$ of health care encounters received by Australians comprised of appropriate, evidence-based care. ${ }^{5}$ To reduce evidence-practice gaps and provide effective preventive care at a population level, primary health care providers must accurately identify patients' health risk status and screening history. Generally, doctors rely on identifying patients' risk status by taking a medical history and referring to medical records; however, these methods may not always identify patients who are at risk. ${ }^{6}$

In Australia, previous studies have indicated there may be poor agreement between patient self-report and data reported by GPs or in medical records, including information on smoking status ${ }^{7}$ and alcohol intake. ${ }^{8}$ In addition, patient recall of screening for cholesterol and cervical cancer may be

\begin{abstract}
Objective: To examine agreement between patients' self-report and general practitioners' perception of their patients' health risk status and screening history.

Methods: Patients attending an Aboriginal Community Controlled Health Service self-reported via survey their health risk status and screening history, while waiting to see their general practitioner (GP). Following the consultation the GP completed a corresponding survey. Prevalence rates and rates of agreement using the kappa statistic were calculated for both self-reported and GP-reported risk status for smoking, at-risk alcohol consumption and physical inactivity; and screening history for blood pressure, cholesterol, diabetes and cervical cancer.

Results: Prevalence rates of health risks were similar from self-report versus GP-reported, yet differed on screening history. Patients who identified themselves as being at risk were often not the same as those identified by GPs. Agreement between patient and doctor was substantial for smoking, yet poor for at-risk alcohol consumption and physical inactivity. Agreement was fair for cholesterol and cervical cancer screening, and slight for blood pressure and diabetes screening.

Conclusions and implications: This study suggests that for effective preventive care, using selfreport for some health risks may be reliable, but less so for screening history. Greater assistance is needed in primary health care settings to identify patients who are at risk.

Key words: Aboriginal health, preventive care, health risks, screening, Aboriginal Community Controlled Health Service
\end{abstract}

inaccurate with patients over-estimating the occurrence of screening. ${ }^{9}$ Yet little is known about agreement between Aboriginal and Torres Strait Islander patients and their doctors on health risk status and screening history. This study examined prevalence of both self-reported and GP-reported risk status for smoking, at-risk alcohol consumption and physical inactivity; and screening history for blood pressure, cholesterol, diabetes and cervical cancer using time intervals recommended by appropriate guidelines. Agreement between patients and their doctors regarding risk status and screening history for each of these measures was also estimated.

\section{Methods}

\section{Ethics and community consultation}

The study was approved by the Human Research Ethics Committee of the University of Sydney, the University of Newcastle and the Western Australian Aboriginal Health Information and Ethics Committee, as well as

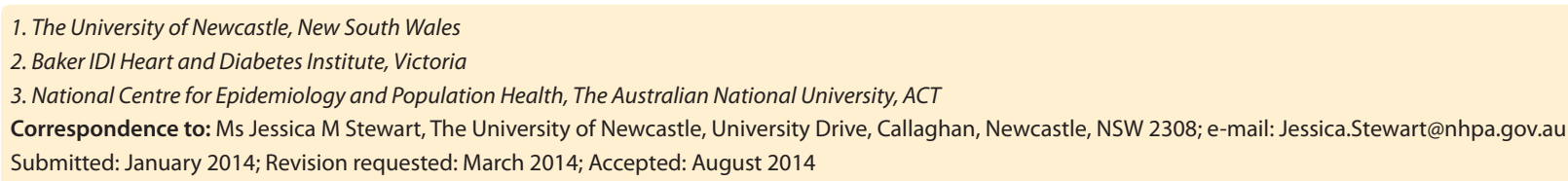


the Board of Management of the Aboriginal Community Controlled Health Service in which the study was based. A local Aboriginal community reference group provided important feedback and advice on cultural and ethical issues regarding the study design, survey content, data collection and analysis.

\section{Setting}

The study was based in a large Aboriginal Community Controlled Health Service with multiple distinct services providing clinical health care and aged care. Aboriginal Community Controlled Health Services are governed by a community-elected board of management and provide holistic health care through an integrated comprehensive primary health care model. ${ }^{10}$ Aboriginal Community Controlled Health Services are independent from the government and represent self-determination in the provision of health care to Aboriginal and Torres Strait Islander people.

\section{Participants}

Participants were recruited in the waiting room as part of a larger survey that collected self-reported information on health risks, screening history and health concerns of Aboriginal patients attending the Aboriginal Community Controlled Health Service. The methods for the larger study are described elsewhere. ${ }^{11}$ Patients were informed that the survey asked about their health and their health risk status, and how often they came to the clinic. They were also informed that their doctor would be asked similar questions at the end of the consultation.

General practitioner sample: All eight general practitioners (GPs) working in any of the three clinics for at least three sessions each per week were approached and agreed to participate in the study.

Patient sample: A sub-sample of patients from the larger survey was obtained using systematic random sampling of every third patient from the appointment list at the start of each randomly selected clinic session. The study planned to recruit 25 surveys from each participating GP providing a sample size of 200. This was determined as feasible with the resources available for the study, while minimising interruptions to the provision of services.

Doctors were informed that the purpose of the study was to examine agreement between patient and doctor on risk status and screening history.

\section{Data Collection}

Four of the research team attended the three clinics over a period of 21 months to recruit patients in the waiting room before their consultation and to assist participants with completion of the survey if necessary. Participants completed a paper questionnaire that was either read out by the research project officer or read by the participant with the interviewer sitting with them, before their scheduled consultation. The treating doctor completed a corresponding survey on the patients' risk status and screening history at the end of the consultation. GPs were notified at the end of the consultation to complete a corresponding survey for the patient.

Definitions of risk categories for each risk factor and optimal screening intervals were derived from the national general practice guidelines for clinical preventive services. 2,12-14 The risk factors and diseases covered in the survey items were selected given their contribution to health outcomes among Aboriginal and Torres Strait Islander people and the potential to effectively reduce the health risks selected in a primary health care setting. ${ }^{9-12}$

The wording of survey items followed that of the current national population survey items and are previously described by Stewart et. al. ${ }^{9}$ and elsewhere. ${ }^{15-17}$ Responses from participants to survey questions categorised them as being 'at risk' if the risk factor was self-reported as being present and 'not at risk' if the risk factor was not present. For smoking, responses of current daily or occasional smoking categorised the patient to be at risk. Responses for alcohol consumption were categorised as at risk if alcohol consumption levels met NHMRC guidelines for at-risk alcohol consumption which equated to no more than four standard drinks on any one occasion for risk of alcohol-related injury, and no more than two standard drinks on any day for lifetime risk of harm from alcohol-related harm. ${ }^{18}$ Alcohol consumption was measured using a seven-day diary recall and the National Health and Medical Research Council of Australia Standard Drinks Chart was shown to participants to help with recall. ${ }^{19}$ Responses for physical inactivity were categorised at being at risk if the response was 'no' to doing at least 30 minutes of physical activity throughout the day on most days of the week.

Responses for screening were categorised as being at risk if the patient self-reported having not been screened within the time interval recommended by appropriate guidelines. Participants were considered at risk if they had not had their blood pressure checked in the previous 12 months, or six months for patients who self-reported having diabetes. For cholesterol, patients were considered at risk if they had not been screened within the previous two years as part of an overall cardiovascular risk status assessment. For diabetes, patients were considered at risk if they were 35 years or older and had not had fasting blood sugar screening within the previous 12 months. Patients who self-reported having type 2 diabetes were excluded from the analysis of patients being at risk of type 2 diabetes. For cervical cancer, women were considered at risk if they were aged between 18 and 69 years and had not had a pap smear in the previous two years, and had not had a hysterectomy.

\section{GP report of patient health risk status and screening history}

After each consultation with a study participant, the treating doctor completed a desk pad page that included the patient's name, age, sex, whether the patient was pregnant, and a rating from one to four of how well they knew the patient. These were matched with the patient's self-reported information. GPs were asked whether the patient was a smoker (yes/no/not sure); and to indicate on a four-point rating scale (from never to always) whether the patient would "in a normal week, drink alcohol above the National Health and Medical Research Council (NHMRC) recommended guidelines?" for long-term 'at risk' alcohol consumption; and "on a single drinking occasion, drink more than the recommended intake of alcohol?" for short-term 'at risk' alcohol consumption. Responses were categorised as either meeting or not meeting recommended guidelines for the purpose of analysis. Responses of never and rarely ( 1 and 2 ) were considered to meet recommended guidelines. Responses of often and always ( 3 and 4) categorised the patient as not meeting guidelines and as being at risk. GPs were asked about whether their patient "in a normal week, does regular physical activity according to recommended guidelines?" Responses of always and often ( 3 and 4) were categorised as meeting recommended guidelines. Responses of never and rarely ( 1 and 2 ) were categorised the patient to be at risk. 
GPs were also asked to tick'yes/no/not sure' or'not applicable' for whether the patient had "Had their blood pressure checked in the last 12 months?"; "Had a cholesterol test in the last 5 years?"; "Had a pap smear in the last 2 years?"; and" Been screened for Diabetes or $\mathrm{HbA1c}$ in the last 3 years?" Doctors were able to consult with their medical records if they considered this appropriate. Responses of 'not sure' or 'don't know' were excluded from the analysis.

\section{Statistical methods}

Statistical analysis was undertaken using STATA version 11. The proportion of patients at risk was obtained with $95 \%$ confidence intervals. GP assessment of patient's risk status was compared to the patient selfreported risk status using the kappa statistic. All kappa values were calculated with $95 \%$ confidence intervals and the $Z$ statistics used to test whether agreement was statistically significantly greater than expected by chance alone. The level of agreement between patient self-report and GP report was classified as follows: $\mathrm{K}<0$ is none/poor; $0 \leq \mathrm{K} \leq 0.20$ is slight; $0.21 \leq k \leq 0.40$ is fair; $0.41 \leq k \leq 0.60$ is moderate; $0.61 \leq k \leq 0.80$ is substantial; and $0.81 \leq \mathrm{k} \leq 1.0$ is almost perfect. ${ }^{20}$

\section{Results}

\section{Patient participation}

A total of 141 participants completed a patient survey for which a doctor completed a corresponding survey. The 141 consenting participants were a sub-sample of a broader patient survey of 587 participants with a consent rate of $77 \% .{ }^{11}$ Forty-four per cent of participants were men and $56 \%$ were women. Nine per cent were aged between 18 and 24 years; $40 \%$ were aged $25-44$ years; $45 \%$ were aged $45-4$ years; and $6 \%$ of participants were 65 years or over. Fourteen responses for smoking status and alcohol consumption were not recorded from participants who declined to answer these questions.

\section{GP participation}

Data were obtained from seven of the eight eligible and consenting doctors. One became ineligible following recruitment due to an unexpected reduction in consultation time at the clinic. Due to the high workload of participating GPs, the number of surveys for individual patients completed by each participating doctor ranged from 11 to 29 with a mean of 20.

\section{Prevalence of health risks and screening history}

Prevalence for smoking, short- and longterm alcohol consumption risk and physical inactivity were similar for estimates based on patients' self-reports, to those of GPs (Table 1). Prevalence of patient self-report for not having been screened within recommended guidelines was generally lower than GPreported prevalence, except for blood pressure, where this relationship was reversed (Table 1). Prevalence estimates were consistent with those found among the larger patient survey population ( $n=587$ ) of which this was a sub-sample (described elsewhere). ${ }^{9}$

\section{Agreement between patients and their treating GP}

Although prevalence was generally similar between patient self-report and GP-report regarding health risk factors, agreement between patients and their treating GP on which individual patients were at risk was generally low for risk factors and for inappropriate screening.

Agreement on health risks: Agreement between patient self-report and GP assessment was substantial for smoking, yet poor for both short-term and long-term harmful alcohol consumption and for physical inactivity.

Agreement on screening history: Agreement between patients and their treating GP regarding who had not been screened within recommended guidelines was fair for cholesterol and cervical cancer screening, yet slight for blood pressure and diabetes screening (Table 2).

\section{Discussion}

The study found similar prevalence reported by patients and GPs of the proportion of patients with health risks, or patients who had not been screened within the recommended time interval for common preventable conditions. With as many as one in four patients not doing sufficient physical activity, this presents substantial opportunity through brief counselling. ${ }^{21}$ In addition, the high prevalence of patients not having been screened within recommended guidelines also presents opportunity for further prevention activities.

Table 1: Prevalence from patient self-report and General Practitioners on patient risk status and screening history. Patients' health risk factor prevalence

\begin{tabular}{lcc} 
& $\begin{array}{c}\text { Patient self-report } \\
\%(95 \% \mathrm{Cl})\end{array}$ & $\begin{array}{c}\text { GP reported } \\
\%(95 \% \mathrm{Cl})\end{array}$ \\
\hline Smoking & $48 \%(40 \%-57 \%)$ & $45 \%(37 \%-54 \%)$ \\
\hline Alcohol (short-term) & $13 \%(8 \%-20 \%)$ & $13 \%(9 \%-20 \%)$ \\
\hline Alcohol (long-term) & $13 \%(8 \%-20 \%)$ & $13 \%(9 \%-21 \%)$ \\
\hline Physical inactivity & $79 \%(71 \%-85 \%)$ & $87 \%(80 \%-91 \%)$ \\
\hline Prevalence of patients not being screened within recommended time intervals according to guidelines \\
\hline
\end{tabular}

Table 2: Agreement between patients and General Practitioners on patient's health risk status and screening

\begin{tabular}{llccc} 
history. & Agreement & Kappa $(95 \% \mathrm{Cl})$ & Records & $P$-value \\
Variables & classification & & & \\
Health risks & Substantial & $0.62(0.48,0.76)$ & 127 & $\mathrm{p}<0.0001$ \\
Smoking & Poor & $-0.01(-0.18,0.16)$ & 127 & $\mathrm{p}<0.9114$ \\
Alcohol (short-term) risk & Poor & $-0.02(-0.18,0.15)$ & 127 & $\mathrm{p}<0.8374$ \\
Alcohol (long-term) risk & Poor & $-0.15(-0.25,-0.05)$ & 141 & $\mathrm{p}<0.0667$ \\
Physical inactivity & & & & \\
\hline Screening & Fair & $0.28(0.12,0.45)$ & 141 & $\mathrm{p}<0.0006$ \\
Cholesterol & Slight & $0.10(-0.05,0.25)$ & 141 & $\mathrm{p}<0.1754$ \\
Diabetes & Slight & $0.02(0.00,0.03)$ & 141 & $\mathrm{p}<0.2818$ \\
Blood pressure & Fair & $0.36(0.15,0.58)$ & 141 & $\mathrm{p}<0.0019$ \\
Cervical cancer & & & & \\
\hline
\end{tabular}


The study found that there was substantial agreement between patients and doctors on the smoking status of patients, ideal for delivering effective evidence-based smoking cessation advice and therapies. 2,2-14,22 There was however poor agreement between patients and doctors regarding patients who were at risk due to alcohol consumption and inadequate physical activity. The low level of agreement between GPs and patients regarding alcohol consumption and physical activity represents opportunity for discussion, and agreement on these behaviours should be achievable between patient and GP.

Only slight and fair agreement on screening for cholesterol, cervical cancer, blood pressure and diabetes may reflect the difficulty patients experience in being able to accurately recall the time since last screening. This highlights the importance of accurate recording of this information on shared patient information systems, such as the personally controlled electronic health record, particularly for patients who attend more than one clinic.

The small sample size, selection bias and social desirability bias are potential limitations to generalising the study findings to other primary health care settings. Social desirability bias, recall bias or the reactive effect on doctors knowing the purpose of the study would have been more likely to underestimate prevalence, and increase agreement, yet prevalence of risk was still high and agreement generally low. The findings of this study are also consistent with other studies on patient/provider agreement in primary care, ${ }^{7-9}$ and with clinical audits in Aboriginal primary health settings that report the need for more complete recording of health risks on patient's medical records. ${ }^{7,23,24}$

\section{Conclusion}

This study shows variation in agreement between patients and doctors on identifying important health risks. Evidence from systematic reviews suggest that the use of standardised practice and feedback is associated with improvements in providing evidence-based care. ${ }^{25,26}$ More routine use of comprehensive risk assessment tools, integrated into electronic patient records, could be an area to target improvements. Many Aboriginal Community Controlled Health Services already actively participate in audit and feedback through quality improvement initiatives to improve the accurate recording of patient information. ${ }^{27-29}$
The study presents preliminary findings that have not previously been reported in the context of Aboriginal and Torres Strait Islander health. Given the large contribution of health risks to the poor health and lower life expectancy experienced by Aboriginal and Torres Strait Islander people, further research is needed to estimate the extent of agreement between patients and doctors, and the opportunities for intervention that may be missed when relying on patient selfreport or incomplete medical records.

Differences in agreement according to patient characteristics could also be considered for further research. Further research is also needed into strategies that encourage more effective doctor-patient partnerships to improve shared risk assessment, health literacy and more patient-directed chronic care risk management. Intervention research studies are also needed to develop evidence of effective prevention strategies that are specifically for Aboriginal and Torres Strait Islander people and communities.

\section{References}

1. Vos T, Barker B, Begg S, Stanley L, Lopez AD. Burden of disease and injury in Aboriginal and Torres Strait Islander Peoples: The Indigenous health gap. Int $J$ Epidemiol. 2009:38(2):470-7.

2. National Association of County and City Health Officials National Guide to a Preventive Health Assessment for Aboriginal and Torres Strait IslanderPeople. 1st ed. South Melbourne (AUST): Royal Australian College of General Practitioners; 2005.

3. Brownson RC, Baker EA, Leet TL, Gillespie KN. Evidencebased Public Health. New York (NY): Oxford University Press; 2003

4. Heywood A, Ring I, Sanson-Fisher R, Mudge P. Screening for cardiovascular disease and risk reduction counselling behaviors of general practitioners. Prev Med. 1994;23(3):292-301.

5. Runciman WB, Hunt TD, Hannaford NA, Hibbert PD, Westbrook Jl, Coiera EW, et al. CareTrack: assessing the appropriateness of health care delivery in Australia. Med J Aust. 2012;197(2):100-5.

6. Panaretto K, Coutts J, Johnson L, Morgan A, Leon D, Hayman N. Evaluating performance of and organisational capacity to deliver brief interventions in Aboriginal and Torres Strait Islander medical services. Aust NZJ Public Health. 2010;34(1):38-44.

7. Dickinson JA, Wiggers J, Leeder SR, Sanson-Fisher RW. General practitioners' detection of patients' smoking status. Med J Aust. 1989;150(8):420-2, 425-6.

8. Reid AL, Webb GR, Hennrikus D, Fahey PP, SansonFisher RW. Detection of patients with high alcohol intake by general practitioners. Br Med J (Clin Res Ed). 1986;293(6549):735-7.

9. Newell S, Girgis A, Sanson-Fisher R, Ireland M. Accuracy of patients'recall of Pap and cholesterol screening. Am J Public Health. 2000;90(9):1431-5.

10. Alford K. Comparing Australian with Canadian and New Zealand Primary Care Health Systems in Relation to Indigenous Populations: Literature Review and Analysis. Discussion Paper No.13. Melbourne (AUST): University of Melbourne Onemda VicHealth Koori Health Unit; 2005.

11. Stewart JM, Sanson-Fisher RW, Eades SJ, D'Este C, Fitzgerald $M$. The risk status, screening history and health concerns of Aboriginal and Torres Strait Islander people attending an Aboriginal Community Controlled Health Service. Drug Alcohol Rev. 2012:31(5):617-24.
12. National Association of County and City Health Officials. Evidence Base to a Preventive Health Assessment in Aboriginal and Torres StraitlslanderPeoples. 1sted. South Melbourne (AUST): Royal Australian College of General Practitioners; 2005.

13. Royal Australian College of General Practitioners. Guidelines for Preventive Activities in General Practice. 6th ed. East Melbourne (AUST): RACGP; 2005.

14. United States Preventive Services Task Force. Pocket Guide to Clinical Preventive Services. Washington (DC): United States Department of Health and Human Services; 2006.

15. Australian Bureau of Statistics. 4715.0 - National Aboriginal and Torres Strait Islander Health Survey. Canberra (AUST): ABS; 2006.

16. Australian Bureau of Statistics. 4713.0.55.001 Populational Characteristics, Aboriginal and Torres Strait Islander Australians. Canberra (AUST): ABS; 2006.

17. Australian Bureau of Statistics. National Aboriginal and Torres Strait/slander Health Survey:Users' Guide, 2004-05. Canberra (AUST): ABS; 2006

18. National Health and Medical Research Council. Australian Alcohol Guidelines: Health Risks and Benefits. Canberra (AUST): NHMRC; 2001.

19. National Health and Medical Research Council. Standard Drinks Chart [Internet]. Canberra (AUST): NHMRC; 2009 [cited 2007 Nov]. Available from: http:// www.nhmrc.gov.au/ files nhmrc/file/your health/ healthy/alcohol/std-drinks.pdf

20. Landis JR, Koch GG. The measurement of observe agreement for categorical data. Biometrics. 1977;33(1):159-74.

21. Orrow G, Kinmonth AL, Sanderson S, Sutton $S$. Effectiveness of physical activity promotion based in primary care: Systematic review and meta-analysis of randomised controlled trials. BMJ. 2012;344:e1389.

22. Stead LF, Bergson G, Lancaster T. Physician advice for smoking cessation (Cochrane Review). In: The Cochrane Database of Systematic Reviews; Issue 2, 2008. Chichester (UK): John Wiley; 2008.

23. Peiris DP, Patel AA, Cass A, Howard MP, Tchan ML, Brady $J$, et al. Cardiovascular disease risk management for Aboriginal and Torres Strait Islander peoples in primary health care settings: Findings from the Kanyini Audit. Med J Aust. 2009;191(6):304-9.

24. Bailie R, Si D, Connors C, Kwedza R, O'Donoghue L, Kennedy $C$, et al. Variation in quality of preventive care for well adults in Indigenous community health centres in Australia. BMC Health Serv Res. 2011;11:139-51.

25. Jamtvedt G, Young JM, Kristoffersen DT, Thomson O'Brien MA, Oxman AD. Audit and feedback: effects on professional practice and health care outcomes Cochrane Review). In: The Cochrane Database of Systematic Reviews; Issue 2, 2006. Chichester (UK): John Wiley; 2006.

26. Rotter T, Kinsman L, James EL, Machotta A, Gothe H, Willis $J$, et al. Clinical pathways: effects on professional practice, patient outcomes, length of stay and hospital costs (Cochrane Review). In: The Cochrane Database of Systematic Reviews; Issue 3, 2010. Chichester (UK): John Wiley; 2010.

27. Panaretto KS, Gardner KL, Button S, Carson A, Schibasaki $\mathrm{R}$, Wason $\mathrm{G}$, et al. Prevention and management of chronic disease in Aboriginal and Islander Community Controlled Health Services in Queensland: A quality improvement study assessing change in selected clinical performance indicators over time in a cohort of services. BMJ Open.2013;3:e002759.

28. Bailie R, Si D, Connors C, Weeramanthri T, Clark L, Dowden M, et al. Study Protocol: Audit and Best Practice for Chronic Disease Extension (ABCDE) Project. BMC Health Serv Res. 2008;8:184

29. Peiris D, Usherwood T, Panaretto K, Harris M, Hun J, Patel B, et al. The Treatment of cardiovascular Risk in Primary care using Electronic Decision support (TORPEDO) study-intervention development and protocol for a cluster randomised, controlled trial of an electronic decision support and quality improvement intervention in Australian primary healthcare. $B M$. Open. 2012;2(6):e002177. 